\title{
Probing general relativity with radar astrometry in the inner solar system
}

\author{
Jean-Luc Margot ${ }^{1}$ and Jon D. Giorgini ${ }^{2}$ \\ ${ }^{1}$ University of California, Los Angeles, \\ 595 Charles Young Drive East, Los Angeles, CA 90095, USA \\ email: jlm@ess.ucla.edu \\ ${ }^{2}$ Jet Propulsion Laboratory, \\ 4800 Oak Grove Drive, Pasadena, CA 91109, USA \\ email: jdg@tycho.jpl.nasa.gov
}

\begin{abstract}
We describe a long-term program designed to obtain and interpret high-precision radar range measurements of a number of near-Earth objects (NEOs) that have trajectories reaching deep inside the gravitational well of the Sun. Objects in our sample have perihelion shift rates 1.5 to 2.5 times that of (1566) Icarus (10"/cy) and span a wide range of inclinations and semi-major axes, allowing for an unambiguous separation of general relativistic and solar oblateness effects. Four objects have been observed at Arecibo on at least two apparitions since 2000 , with typical uncertainties of a few hundred meters. Within the next three years, we anticipate securing a total of 15 observations of 5 different NEOs. This program is expected to provide a purely dynamical measurement of the oblateness of the Sun $\left(J_{2}\right.$ at the $10^{-8}$ level $)$ and to constrain the Eddington parameter $\beta$ at the $10^{-4}$ level. Although our objects are selected to minimize Yarkovsky orbital drift, we also anticipate measuring Yarkovsky drift rates, which are orthogonal to the GR and $J_{2}$ signatures.
\end{abstract}

Keywords. general relativity, solar quadrupole moment, Yarkovsky drift, asteroids, radar

\section{Motivation}

Attempts to quantize gravity and to unify it with other forces indicate that general relativity (GR) cannot be the final theory on gravity (Will 2006). Testing metric theories of gravity to higher levels of precision is, therefore, critical and has resulted in new efforts in the solar system (e.g. Nordtvedt 2000; Margot 2003; Pireaux and Rozelot 2003; Iorio 2005; Folkner 2009). While the uncertainty on $\gamma$ in the parametrized post-Newtonian (PPN) formalism is now of order $10^{-5}$ (Bertotti et al. 2003), there has been no comparable improvement in the knowledge of $\beta$. A direct constraint on $\beta$ can be obtained by measurement of the perihelion shift. Anderson et al. (2002) have combined radar and spacecraft ranging data and find $|\beta-1|<1.2 \times 10^{-3}$, while Folkner (2009) recently reported $|\beta-1|<10^{-3}$. Our simulations show that Arecibo radar measurements obtained over a decade can discriminate changes in $\beta$ at the $10^{-4}$ level.

A second motivation for our observations stems from the difficulties in reconciling helioseismological inferences with new solar abundance measurements. Confidence in the helioseismology inversions has been shaken as independent solar abundance measurements displaying a high degree of consistency (Asplund et al. 2004; Caffau et al. 2008) have ruined the previous agreement between helioseismological inferences and models of the solar interior. The new measurements place the oxygen abundance at $\sim 60 \%$ of the Anders and Grevesse (1989) values, changing the opacity and depth of the base of the convective layer (Basu and Antia 2008). The quadrupole moment of the Sun is of fundamental importance to the internal structure of the Sun and warrants an independent 
determination that does not rely on inversion models of helioseismology data. Our simulations show that changes in the solar quadrupole moment $J_{2}$ at the $10^{-8}$ level are detectable and would put the preferred helioseismology value of $J_{2} \sim 2 \times 10^{-7}$ (Pireaux and Rozelot 2003) to a very serious test with a direct dynamical measurement.

Finally we are motivated by the benefits of measuring the Yarkovsky orbital drift, which is due to the anisotropic reradiation of sunlight from asteroid surfaces (Bottke et al. 2006). This effect has been detected with Arecibo radar data (Chesley et al. 2003) and turns out to be the dominant source of uncertainty in near-Earth asteroid (NEA) trajectory predictions (Giorgini et al. 2002) for bodies smaller than $2 \mathrm{~km}$. By far the largest influence on orbital parameters is a change in the semi-major axis of objects as a function of their spin, shape, orbit, and material properties. For asteroids of known sizes and spins, a measurement of the Yarkovsky drift rate can be interpreted in terms of bulk density and thermal properties (Chesley et al. 2003). Our goal is to obtain such measurements along with detailed physical characterizations, with a particular focus on the binaries in our sample for which independent density estimates can be established.

\section{Theoretical background}

The spacetime geometry around a spherical star is described by a metric that is static and spherically symmetric (Schwarzschild 1916). In isotropic coordinates,

$$
d s^{2}=-\left(1-2 \frac{G M}{c^{2} r}+2\left(\frac{G M}{c^{2} r}\right)^{2}\right)(c d t)^{2}+\left(1+2 \frac{G M}{c^{2} r}\right)\left[d x^{2}+d y^{2}+d z^{2}\right],
$$

where $G$ is the gravitational constant, $c$ is the speed of light, and $M$ is the mass of the star. GR derives part of its elegance from the fact that it depends only on $G$ and $c$, which are non-adjustable constants.

The PPN formalism is a framework to parametrize various theories of gravity in a systematic way. Of the ten parameters, $\beta$ and $\gamma$ are the most important. Their placement in the metric illuminates their physical significance:

$$
d s^{2}=-\left(1-2 \frac{G M}{c^{2} r}+2 \beta\left(\frac{G M}{c^{2} r}\right)^{2}\right)(c d t)^{2}+\left(1+2 \gamma \frac{G M}{c^{2} r}\right)\left[d x^{2}+d y^{2}+d z^{2}\right] .
$$

Appearing in the spatial part of the metric, $\gamma$ is related to the amount of curvature produced by a unit rest mass, and is tested by deflection of light and Shapiro delay experiments. The degree of non-linearity in the superposition law for gravity is captured by $\beta$. In GR, $\beta=\gamma=1$.

Orbits of test particles in curved spacetime do not close and their perihelion precesses. A Keplerian orbit modified for perihelion precession can be written (Misner et al. 1973)

$$
r=\frac{a\left(1-e^{2}\right)}{1+e \cos [(1-\delta \phi / 2 \pi) \phi]}
$$

where $a$ is the semi-major axis, $e$ is the eccentricity, and $\phi$ is the true anomaly. The perihelion shift per orbit is

$$
\delta \phi=\frac{6 \pi G M_{\odot}}{a\left(1-e^{2}\right) c^{2}} \frac{(2-\beta+2 \gamma)}{3} .
$$

Under the influence of an oblate Sun with quadrupole moment $J_{2}$, the perihelion shift 
contains an additional contribution:

$$
\delta \phi=\frac{6 \pi G M_{\odot}}{a\left(1-e^{2}\right) c^{2}}\left[\frac{(2-\beta+2 \gamma)}{3}\right]+\frac{6 \pi}{2} R_{\odot}^{2} \frac{\left(1-3 / 2 \sin ^{2} i\right)}{a^{2}\left(1-e^{2}\right)^{2}} J_{2},
$$

with $i$ the orbital inclination with respect to the solar equator and $R_{\odot}$ is the solar radius.

\section{Previous studies}

Shapiro et al. [1968, 1971, 1972] determined the perihelion precession of Mercury and that of asteroid (1566) Icarus (Gilvarry 1953) to test GR and to constrain values of the PPN parameters. Because Newtonian precession due to the oblate Sun affects the measurements, Shapiro emphasized the need to measure the precession of several bodies in order to separate, based on their heliocentric distance dependence, the general relativistic effects from those due to the gravitational quadrupole moment of the Sun $\left(J_{2}\right)$. Another way of separating the two effects is to use several bodies with different orbital inclinations, since GR is a purely central effect whereas the precession due to the oblate Sun has a known dependence on inclination. The GR and $J_{2}$ influences cause no change in the semi-major axis, which is orthogonal to the effect of the Yarkovsky drift . We rely on these different signatures on orbital evolution to distinguish the Yarkovsky effect from the perihelion shift.

The perihelion shift of Mercury predicted from GR alone is 43 arcseconds per century ("/cy) (Nobili and Will 1986). The value measured with radar is known with $0.5 \%$ uncertainties (Shapiro et al. 1976; Anderson et al. 1991) and is consistent with GR predictions. The influence of the solar $J_{2}$ has not been detected and is $\sim 0.1 \%$ of the GR influence for $J_{2} \sim 2 \times 10^{-7}$.

We anticipate improvements over previous studies involving Mercury and Icarus because 1) Several newly-discovered asteroids have orbits offering a better sensitivity to the solar $J_{2} ; 2$ ) Our sample incorporates a range of heliocentric distances and inclinations that can unambiguously separate GR and $J_{2}$ effects and provide more robust estimates in a joint solution; 3 ) The center of mass locations of the small bodies can be determined to 100-500 m, about an order of magnitude better than existing Mercury ranges. The Mercury determinations suffer from km-scale uncertainties due to unknown topography and possible center of mass/center of figure offset. Mercury topography is an important source of systematic errors in GR tests (Pitjeva 1993).

\section{Observational strategy}

In light of the large number of recent NEO discoveries, a search for asteroids that provide better opportunities than Mercury or Icarus to detect GR and $J_{2}$ effects was performed (Margot 2003). Roughly ten candidates with long astrometric arcs, repeated observability at Arecibo, and GR perihelion shifts larger than that of Icarus have been identified (Figure 1 and Table 1). We regularly update the target list to incorporate the NEOs most suited to the realization of our science objectives.

We rely primarily on optical astrometry (typically hundreds of measurements) to secure state estimates for each object, and we rely primarily on the radar measurements to expose the parameters of interest: one Yarkovsky rate per object, $\beta$, and $J_{2}$.

As shown in Table 1, we have now acquired observations on two apparitions for 4 objects (1999 KW4, 1999 MN, 2000 BD19, 2000 EE14), giving very roughly 8 independent data constraints for 6 solve-for parameters (In reality, six orbital parameters must also be determined - see previous paragraph). In the next three years a modest investment 

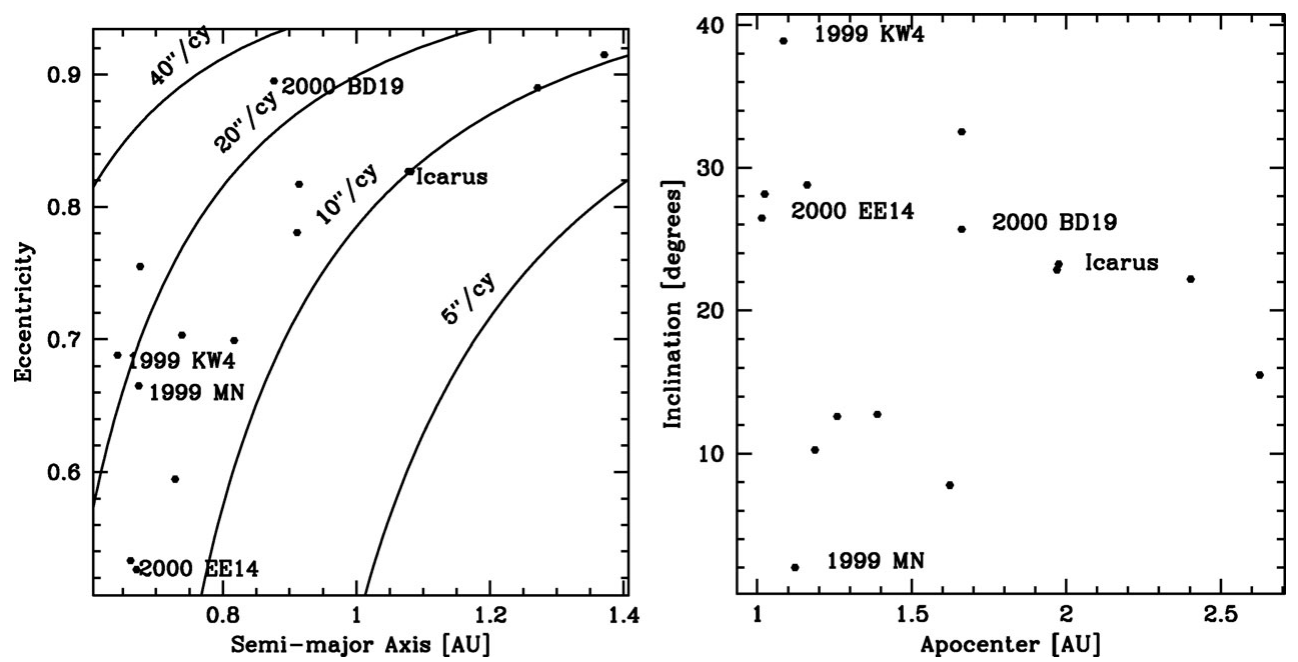

Figure 1. A. Predicted rates of perihelion shift due to GR alone for a number of newly discovered NEAs, compared to that of (1566) Icarus, shown in semi-major axis versus eccentricity space. Objects with two existing radar detections are labeled. B. Same objects shown in apocenter versus inclination space, illustrating the wide range of inclinations that can separate GR and $J_{2}$ effects.

\begin{tabular}{|c|c|c|c|c|c|c|c|c|c|c|c|}
\hline Target & $\mathrm{H}$ & $\begin{array}{r}\mathrm{D} \\
{[\mathrm{km}]}\end{array}$ & $\begin{array}{r}\mathrm{P} \\
{[\mathrm{h}]}\end{array}$ & $\begin{array}{r}\text { Arc } \\
\text { days] }\end{array}$ & $N_{\mathrm{o}}$ & $N_{\mathrm{r}}$ & $\begin{array}{r}a \\
{[\mathrm{AU}]}\end{array}$ & $e$ & $\begin{array}{r}i \\
{[\mathrm{deg}]}\end{array}$ & $\begin{array}{r}\dot{\tilde{\omega}} \\
(" / \mathrm{cy})\end{array}$ & App \\
\hline & & & & & & 2 & & & & & \\
\hline & & & & & & 2 & & & & & \\
\hline & & & & & & 2 & & & & & \\
\hline & & & 5. & & & 2 & 0 & & & & \\
\hline 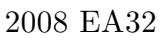 & & 2.1 & 5.0 & & 6 & & 0 & & & 4.2 & 1112 \\
\hline & J.5 & & 2.3 & 20 & & 1 & 1.078 & & 2.0 & 0.1 & \\
\hline Phaethon & 14.6 & 5.10 & 3.6 & 9231 & 1801 & 1 & 1.271 & & 22.2 & 10.1 & $\begin{array}{lll}13 & 16 & 17\end{array}$ \\
\hline alos & 17.0 & 1.60 & 38.5 & 6234 & 415 & & 1.081 & 0.827 & 23.2 & 10.0 & 1011 \\
\hline
\end{tabular}

Table 1. Subset of NEOs that are particularly well-suited for our program, based on the NEO population known as of 2009 May $30 . \mathrm{H}$ is the absolute magnitude, $\mathrm{D}$ is diameter, and $\mathrm{P}$ is spin period. Sizes and spin periods were obtained from the DLR NEA Data Base, unless superseded by our own radar estimates (in bold). For those objects that had no size/albedo information, the value was evaluated on the basis of $\mathrm{H}$ and the $11 \%$ average albedo of NEAs (italics). For those objects that had no spin period information, the period was fixed at 5 hours and italicized in the table. Arc and $N_{\mathrm{o}}$ refer to the interval between first and last optical observation and the total number of optical observations, respectively. All objects have arc lengths in excess of 300 days, guaranteeing recovery and small pointing uncertainties. $N_{\mathrm{r}}$ is the number of apparitions with existing ranging observations. Orbital elements $a, e, i$ have their usual definition. The perihelion shift rate $\dot{\tilde{\omega}}$ is given in arcseconds per century, with a cutoff of $10 " /$ cy. The last column indicates the years of future apparitions detectable at Arecibo.

of $\sim 70$ hours of telescope time can secure ranges for an additional 7 epochs (Table 1 ). In rough accounting terms, there will be a total of 15 independent data constraints (for 5 different objects) and 7 solve-for parameters. At least three objects will have data on at least three apparitions, giving good prospects for measuring Yarkovsky drift rates. Yarkovsky drift rates are roughly $15 \mathrm{~m} / \mathrm{y}$ in semi-major axis for a $1 \mathrm{~km}$ object, and the rate scales roughly as size ${ }^{-1}$. The range is affected quadratically with time and rapidly produces a signal of several $\mathrm{km}$ (Chesley et al. 2003). 
We obtain accurate range astrometry to NEAs using procedures that have been refined to exquisite precision over the years. The basic idea is to send a waveform encoded with a pseudo-random code sequence and to cross-correlate the received echoes with a replica of the code (Evans and Hagfors 1968). Transmission occurs for the duration of the round-trip light time to the object, and reception occurs for an equivalent duration. Each transmit-receive cycle constitutes a run. The duration of an individual code element (baud) and the length of the code are chosen in combinations that provide unambiguous range measurements to distances of several astronomical units. So-called closed-loop tests are performed with identical system parameters to fully calibrate delays within the telescope and electronics. The internal consistency of the measurements and external verifications via orbit determination software are both excellent.

Figure 2 illustrates that for ranging uncertainties of $\sim 100 \mathrm{~m}$, a $10^{-3}$ variation in $\beta$ represents a 10- $\sigma$ signal after a decade with a single NEO. Our goal is to constrain $\beta$ at the $10^{-4}$ level (and $J_{2}$ at the $10^{-8}$ level) from a joint analysis of the entire data set.

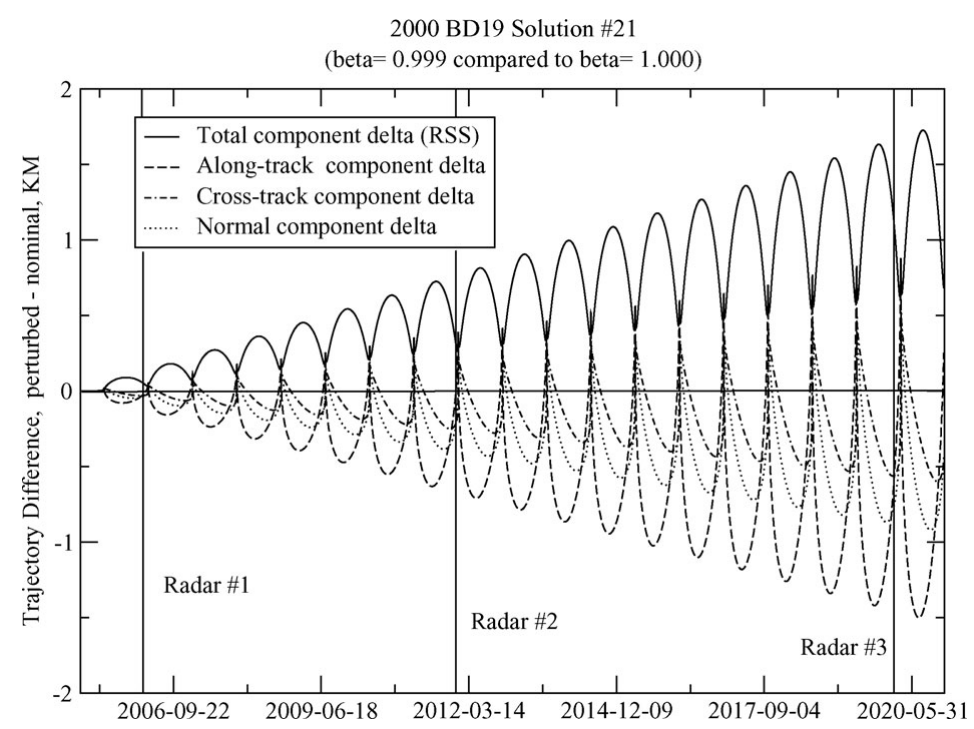

Figure 2. Sensitivity of the trajectory of NEO 2000 BD19 to variations in $\beta$ at the $10^{-3}$ level.

\section{Conclusions}

The Yarkovsky and perihelion shift observations represent a long-term endeavor with little or no instant gratification. However, a modest investment in telescope time can improve our knowledge of asteroid densities and thermal properties, provide a dynamical measurement of the solar $J_{2}$, and test general relativity to new levels of precision.

\section{References}

E. Anders and N. Grevesse. Abundances of the elements - Meteoritic and solar. Geochim. Cosmochim. Acta, 53:197-214, January 1989.

J. D. Anderson, M. A. Slade, R. F. Jurgens, E. L. Lau, X. X. Newhall, and E. M. Standish. Radar and spacecraft ranging to Mercury between 1966 and 1988. Proceedings of the Astronomical Society of Australia, 9:324-+, 1991.

J. D. Anderson, E. L. Lau, S. Turyshev, J. G. Williams, and M. M. Nieto. Recent Results for Solar-System Tests of General Relativity. Bulletin of the American Astronomical Society, 34:660-+, May 2002. 
M. Asplund, N. Grevesse, A. J. Sauval, C. Allende Prieto, and D. Kiselman. Line formation in solar granulation. IV. [O I], O I and $\mathrm{OH}$ lines and the photospheric $\mathrm{O}$ abundance. A\&A, 417:751-768, April 2004.

S. Basu and H. M. Antia. Helioseismology and solar abundances. Phys. Rep., 457:217-283, March 2008.

B. Bertotti, L. Iess, and P. Tortora. A test of general relativity using radio links with the Cassini spacecraft. Nature, 425:374-376, September 2003.

W. F. Bottke, Jr., D. Vokrouhlický, D. P. Rubincam, and D. Nesvorný. The Yarkovsky and Yorp Effects: Implications for Asteroid Dynamics. Annual Review of Earth and Planetary Sciences, 34:157-191, May 2006.

E. Caffau, H.-G. Ludwig, M. Steffen, T. R. Ayres, P. Bonifacio, R. Cayrel, B. Freytag, and B. Plez. The photospheric solar oxygen project. I. Abundance analysis of atomic lines and influence of atmospheric models. A\&A, 488:1031-1046, September 2008.

S. R. Chesley, S. J. Ostro, D. Vokrouhlický, D. Čapek, J. D. Giorgini, M. C. Nolan, J. Margot, A. A. Hine, L. A. M. Benner, and A. B. Chamberlin. Direct Detection of the Yarkovsky Effect by Radar Ranging to Asteroid 6489 Golevka. Science, 302:1739-1742, December 2003.

John V. Evans and Tor Hagfors, editors. Radar Astronomy. McGraw-Hill, New York, 1968.

W. M. Folkner. Relativistic Aspects of the JPL Planetary Ephemeris. American Astronomical Society, IAU Symposium \#261, 155. Relativity in Fundamental Astronomy: Dynamics, Reference Frames, and Data Analysis 27 April - 1 May 2009 Virginia Beach, VA, USA, \#6.01, 261, May 2009.

J. J. Gilvarry. Relativity Precession of the Asteroid Icarus. Physical Review, 89:1046-1046, March 1953.

J. D. Giorgini, S. J. Ostro, L. A. M. Benner, P. W. Chodas, S. R. Chesley, R. S. Hudson, M. C. Nolan, A. R. Klemola, E. M. Standish, R. F. Jurgens, R. Rose, A. B. Chamberlin, D. K. Yeomans, and J.-L. Margot. Asteroid 1950 DA's Encounter with Earth in 2880: Physical Limits of Collision Probability Prediction. Science, 296:132-136, April 2002.

L. Iorio. On the possibility of measuring the solar oblateness and some relativistic effects from planetary ranging. A\&A, 433:385-393, April 2005.

J. L. Margot. Candidate Asteroids for Discerning General Relativity and Solar Oblateness. AAS/Division of Dynamical Astronomy Meeting, 34, August 2003.

Charles W. Misner, Kip S. Thorne, and John Archibald Wheeler. Gravitation. Freeman, 1973.

A. M. Nobili and C. M. Will. The real value of Mercury's perihelion advance. Nature, 320:39-41, March 1986.

K. Nordtvedt. Improving gravity theory tests with solar system "grand fits". Phys. Rev. D, 61 (12):122001-+, June 2000.

S. Pireaux and J.-P. Rozelot. Solar quadrupole moment and purely relativistic gravitation contributions to Mercury's perihelion advance. Ap\&SS, 284:1159-1194, 2003.

E. V. Pitjeva. Experimental testing of relativistic effects, variability of the gravitational constant and topography of Mercury surface from radar observations 1964-1989. Celestial Mechanics and Dynamical Astronomy, 55:313-321, April 1993.

K. Schwarzschild. On the Gravitational Field of a Mass Point According to Einstein's Theory. Abh. Konigl. Preuss. Akad. Wissenschaften Jahre 1906,92, Berlin,1907, pages 189-196, 1916.

I. I. Shapiro, M. E. Ash, and W. B. Smith. Icarus - Further Confirmation of Relativistic Perihelion Precession. Physical Review Letters, 20(26):1517+, 1968.

I. I. Shapiro, W. B. Smith, M. E. Ash, and S. Herrick. General Relativity and the Orbit of Icarus. Astron. J., 76:588-+, September 1971.

I. I. Shapiro, R. P. Ingalls, R. B. Dyce, M. E. Ash, D. B. Campbell, and G. H. Pettengill. Mercury's Perihelion Advance - Determination by Radar. Physical Review Letters, 28(24): $1594+, 1972$.

I. I. Shapiro, C. C. Counselman, and R. W. King. Verification of the principle of equivalence for massive bodies. Physical Review Letters, 36:555-558, March 1976.

C. M. Will. The Confrontation between General Relativity and Experiment. Living Reviews in Relativity, 9:3-+, March 2006. 